\title{
ANTHROPEN
}

Le dictionnaire francophone d'anthropologie ancré dans le contemporain

\section{MÈRES PORTEUSES}

De Koninck, Maria

Université Laval, Canada

Date de publication : 2021-10-05

DOI: https://doi.org/10.47854/anthropen.v1i1.51292

Voir d'autres entrées dans le dictionnaire

On appelle mère porteuse une femme qui, en vertu d'une entente avec une personne seule ou un couple, s'engage à porter pendant neuf mois un enfant qu'elle lui cédera après l'avoir mis au monde.

Le recours aux mères porteuses s'inscrit dans le contexte du développement de la procréation médicalement assistée (PMA). Facilité par la fécondation in vitro (FIV), il s'est multiplié à la fin du siècle dernier. (Voir Procréation médicalement assistée).

Dans les premiers cas rapportés, la mère porteuse était inséminée, la fécondation ayant lieu in vivo. Cette façon de faire, qualifiée de "traditionnelle ", a progressivement cédé la place à la fécondation in vitro. Un embryon, créé à partir de l'ovule d'une autre femme et du sperme du demandeur (ou spermes mélangés des demandeurs) pour assurer un lien génétique paternel, est implanté chez la mère porteuse (CEST 2009). La maternité est ainsi répartie entre trois femmes, la mère génétique (qui a fourni l'ovule), la mère biologique (qui porte l'enfant) et la mère sociale (qui reçoit l'enfant).

L'entente entre la mère porteuse et le ou les demandeurs peut être informelle, notamment dans le cas de membres d'une même famille. Le plus souvent, elle est formelle et un contrat est signé entre les deux parties. En l'absence de rémunération, cette pratique sociale est qualifiée «d'altruiste » et seules les dépenses encourues sont couvertes par le ou les demandeurs. Quand la mère porteuse est rémunérée, l'entente est qualifiée de «commerciale » (CSF 2016). Le contrat n'a de valeur légale que lorsque l'entente a été conclue dans une juridiction qui n'interdit pas cette pratique. 
Utilisée pour contrer certaines formes d'infertilité ou d'incapacité à se reproduire chez des couples hétérosexuels, la pratique est devenue une réponse à l'incapacité de se reproduire, attribuable à des situations sociales telles que le célibat masculin ou l'union entre deux hommes, participant ainsi du processus de médicalisation du social (Conrad 2007). Elle est, de plus, devenue une « solution de convenance » pour des femmes ne souhaitant pas porter leur enfant, comme le rapporte la presse du divertissement (Loulier 2009).

Le nombre de termes utilisés pour désigner les acteurs et décrire la pratique est élevé. Des chercheuses anglophones ont recensé 23 façons de nommer les femmes qui acceptent de céder un enfant après l'avoir porté, 13 pour nommer les personnes qui font appel à elles, 5 pour celles qui fournissent des ovules, 6 pour ceux qui fournissent du sperme et 20 pour la pratique (Beeson, Darnovsky et Lippman 2015). La majorité de ces termes ont une correspondance en français.

Cette diversité des noms témoigne des multiples angles sous lesquels est abordé le rôle des mères porteuses (mère biologique, gestatrice, contractuelle, travailleuse, hôte), celui des futurs parents (intentionnels, commanditaires, contractuels, sociaux, adoptifs), des personnes offrant ovules ou sperme (fournisseurs, donneuses ou donneurs, mère ou père génétique), et de la façon dont est décrite la pratique elle-même (manifestation d'altruisme, affaire, grossesse ou maternité sous contrat, enfantement ou gestation pour autrui, maternité de substitution, reproduction avec un tiers).

En français, le concept le plus utilisé est celui de gestation pour autrui (GPA). Or, le mot "gestation » est habituellement réservé au monde animal, le terme grossesse étant privilégié pour désigner l'expérience féminine. Maternité de substitution est aussi fréquemment utilisé, la grossesse et la mise au monde désignées de cette façon référant à une expérience maternelle vécue seulement à titre de ressource substitut. En langue anglaise, le terme usuel pour désigner la pratique du recours aux mères porteuses est surrogacy et pour les mères porteuses surrogates (substituts). L'accent est ainsi mis sur cette acception de la signification de la grossesse et de l'accouchement comme pouvant être un service offert dans le contexte d'une entente. Enfin, on trouve aussi l'expression tierce reproductrice pour qualifier la mère porteuse. Cette appellation trouve sa source dans une représentation de la procréation à plusieurs comme façon de construire les liens de parenté en dehors du modèle de mère unique (Côté, Lavoie et Courduriès 2018).

Les termes désignant les parents qui ont recours aux mères porteuses nous renseignent sur les indications justifiant l'utilisation de la PMA. Ces indications étaient, à l'origine, essentiellement médicales. Or, les futurs parents ayant recours à la PMA sont dorénavant de plus en plus souvent appelés «parents d'intention » ayant développé un «projet parental », soit celui de se reproduire (Comité consultatif sur le droit de la famille 2015). Cette évolution amène à justifier le recours à une tierce personne par le souhait d'avoir un enfant et même, éventuellement, de revendiquer le droit à l'enfant, droit qui n'a aucun fondement 
juridique (Brunetti-Pons et al. 2017). L'autre appellation d'utilisation connue d'usage fréquent est celle de "parents commanditaires ", qui met plutôt l'accent sur le caractère transactionnel de cette forme de procréation en référant à l'existence d'un contrat (PDF Québec 2017).

À l'échelle de la planète, devant cette pratique, les pays adoptent l'une des quatre positions suivantes : interdiction ; aucune reconnaissance légale mais pas d'interdiction ; reconnaissance légale, accompagnée d'un encadrement plus ou moins sévère, de la seule pratique "altruiste » ou des pratiques " altruiste » et « commerciale »; laxisme total. À l'intérieur d'un même pays, des différences marquées entre États, provinces ou juridictions peuvent exister (HRC 2018). C'est le cas du Canada où la pratique « altruiste » est permise, alors qu'au Québec, la validité d'un contrat entre une mère porteuse et un ou des demandeurs n'est pas reconnue. Aux États-Unis, il y a de tout, l'éventail allant de l'interdiction au laxisme total (Garrison 2014).

Le statut légal de la pratique des mères porteuses est en constante évolution (Surrogacy 360.0rg). Certains pays d'abord permissifs, le cas le plus célèbre étant celui de l'Inde, ont resserré les contrôles, le plus souvent en limitant l'accès aux mères porteuses à leurs seuls citoyens. Lorsque les pays procèdent ainsi, d'autres, en général plus défavorisés, prennent la relève, à l'instar de ce qui s'observe dans le déplacement des industries multinationales. Ailleurs, où la pratique n'est pas autorisée ou plus contraignante, notamment en Europe et au Canada, les conditions évoluent dans le sens contraire. Des demandeurs ramènent des enfants de pays permissifs et, par l'obtention de décisions juridiques en leur faveur, provoquent des brèches dans les interdictions ou contraintes existantes - ce que la juriste Marie-Anne Frison-Roche décrit comme une « façon d'éviter le pouvoir du législateur pour séduire le pouvoir juridictionnel » (2016).

Le recours aux mères porteuses interpelle nécessairement de nombreuses disciplines, dont l'anthropologie, car il concerne la reproduction et par conséquent les rapports sociaux de sexe, la filiation et la parenté. En introduisant une tierce personne dans la reproduction, il soulève des questions fondamentales pour toute société et suscite la polémique. Parmi ces questions on note : l'utilisation d'un être humain à des fins de reproduction ; la remise en question du concept de mère, mater semper certa est, comme étant nécessairement la femme qui accouche ; la possibilité pour des individus et des couples d'obtenir un enfant de façon contractuelle ; l'embrouillement des règles établies en matière de filiation; le tourisme procréatif, dans des pays à faible revenu, auquel elle donne lieu en tant que pratique sans frontières. Tout cela explique que la légitimité de cette façon de se reproduire n'obtienne pas de consensus et que les débats soient vifs dans plusieurs sociétés, particulièrement en Europe.

Une question centrale en anthropologie est de savoir si le recours à une femme pour porter un enfant qu'elle cédera à sa naissance marque une rupture avec les pratiques connues en reproduction humaine ou s'il n'en est qu'une forme contemporaine. Certains anthropologues considèrent que celui-ci s'inscrit bien 
dans l'évolution en matière de reproduction (Courduriès 2016) alors que Godelier (2014) écrit n'avoir jamais observé une telle façon de faire. Selon Françoise Héritier (2010:35), c'est l'introduction du critère de vérité génétique qui pose problème, car sa recherche présente le danger d'une "société sans recours à l'altérité pour créer du lien social ». Ces différentes considérations induisent des questions sur les règles définissant la parenté et la filiation (Feuillet-Liger et Crespo-Bauner 2014 ; Gunnarsson Payne 2016).

Du côté des milieux favorisant le recours aux mères porteuses, l'exemple le plus fréquemment utilisé pour ancrer cette pratique dans l'histoire de l'humanité est tiré de l'Ancien Testament. II s'agit de l'histoire de Hagar, servante de Sarah qui était infertile, qui a donné un enfant à Abraham, époux de sa maîtresse. Revient également l'exemple du don d'enfant, don usuel dans certaines cultures, telles celles de certaines communautés autochtones. Cet exemple est toutefois cité sans précisions sur les contextes culturels ni sur les caractéristiques de ce type de dons qui peuvent correspondre à une pratique d'adoption informelle (Decaluwe, Poirier et Muckle 2016).

Les recherches ethnographiques menées auprès de mères porteuses, de demandeurs, d'intermédiaires et de membres de cliniques de PMA sont nombreuses. Dès 1994, Helena Ragone a publié les résultats d'une vaste étude menée auprès de mères porteuses. À ce chapitre, l'Inde a été un terrain de recherche fertile, car des mères porteuses ont pu être jointes dans les cliniques où elles étaient confinées pendant leur grossesse. Des travaux, réalisés dans ce pays et ailleurs, permettent de mieux comprendre leurs expériences. Les femmes interviewées ont décrit leurs réactions devant la perspective d'avoir à céder un enfant porté et leur sentiment de capacitation attribuable, entre autres, au revenu tiré de la pratique. Elles ont parlé du sens qu'elles accordaient à leur contribution au projet d'autres personnes, soit celui de pouvoir faire un don et de créer du bonheur. Elles ont aussi partagé leurs inquiétudes, exposé les difficultés de vivre les contraintes et souligné leurs efforts de ne pas s'attacher aux enfants portés (Harrison 2014 ; Jacobson 2016 ; Saravan 2018).

Les propos recueillis auprès des demandeurs nous informent sur leurs justifications pour recourir à une tierce personne: la quête de la fertilité et la volonté d'avoir un lien génétique avec l'enfant, ce qui exclut l'adoption ; la légitimité de leur démarche, étant donné l'évolution des mœurs et du concept de famille ; le peu d'importance accordée à la grossesse et à l'enfantement. Des témoignages révèlent également que les demandeurs ressentent le besoin de se disculper et considèrent bien traiter les mères porteuses (Gross 2018).

Du côté des études féministes, il y a divergence (Bandelli 2019 ; Corradi 2020). Certaines auteures présentent cette pratique comme une manifestation d'autonomie, les femmes pouvant décider que faire avec leur corps, considérant qu'il s'agit d'une façon de s'émanciper et d'une reconnaissance du travail reproductif dans le cas de celles qui sont rémunérées (lacub 2004 ; Noel 2015). D'autres auteures l'interprètent plutôt comme une appropriation du potentiel 
reproducteur des femmes, une atteinte à leur dignité humaine, une négation de la dimension relationnelle de l'expérience de la grossesse et de l'accouchement, un discours abusif sur les femmes et le don, ainsi qu'une exploitation de femmes démunies, notamment dans le cas de celles issues de pays à faible revenu (Agacinski 2013 ; Klein 2017 ; Segalen 2017 ; Guilbault 2018).

Plusieurs travaux portent sur les écueils éthiques soulevés par une telle pratique en ce qui concerne, entre autres, la liberté, la dignité humaine, les droits humains des femmes et des enfants, et l'inviolabilité du corps humain (Agacinski 2013 ; Segalen 2017 ; De Koninck 2019). D'autres s'attardent aux principes déontologiques mis en cause par les interventions médicales, à savoir l'autonomie (Épelboin 2011), le risque de malveillance puisque l'incidence de complications pour la mère et l'enfant est plus élevée que lors d'une grossesse spontanée (Allen 2018), et le fait que les soins prodigués à une personne ne le soient pas dans son propre intérêt, mais bien pour répondre à la demande de tiers (Brunetti-Pons 2017).

La forme «commerciale » du recours aux mères porteuses est celle qui soulève davantage de critiques. En voici quelques exemples :

a) Céline Lafontaine utilise le concept de bioéconomie pour traiter d'interventions faisant un "usage biotechnologique des éléments corporels en tant que ressources premières pour l'industrie biomédicale " (2014: 12) et qualifie l'utilisation de mères porteuses d'exemple «le plus extrême du processus de sous-traitance du travail reproductif » (2014:188) ;

b) au cœur de la pratique du recours aux mères porteuses se trouve l'industrie de la PMA, dont les profits sont très élevés, le nombre de cliniques se multipliant comme celui des acteurs - médecins et autres intervenants de la santé, intermédiaires, juristes, etc. Ekman (2017) établit, à l'instar d'autres chercheuses, une comparaison avec le marché de la prostitution ;

c) une analyse de contrats signés en Californie a permis de constater l'étendue des droits obtenus par des demandeurs sur la vie de mères porteuses (Lahl 2018) ;

d) des parents ont refusé de recevoir un enfant handicapé et l'ont laissé à la charge de la mère porteuse, démontrant le risque inhérent à cette pratique dans certains contextes (Tourame 2016).

Enfin, dans les études et les discours portant sur la pratique du recours aux mères porteuses, on accorde peu d'égards à l'enfant, raison d'être de la procréation, cet " otage invisible de décisions qui pèseront sur son existence tout entière » (Le Breton 2014). L'intérêt est généralement centré sur l'expérience des demandeurs, des mères porteuses et des acteurs entourant ces derniers. Pourtant l'enfant a des droits et ne peut faire l'objet d'un échange ; le débat sur le risque que cette pratique soit, dans les faits, une forme de «vente d'enfant » est loin ISSN : 2561-5807, Anthropen, Université Laval, 2021. Ceci est un texte en libre accès diffusé sous la licence CC-BY-NCND, https://creativecommons.org/licenses/by-nc-nd/4.0/ 
d'être tranché (HRC 2018). Même si les tenants de la pratique postulent que les enfants bien entourés à la naissance s'en tireront sans problèmes identitaires ou affectifs, les préoccupations concernant les impacts sur un enfant d'avoir été séparé à la naissance de la femme qui l'a porté et mis au monde n'ont pas trouvé de réponse. La question se pose : si l'enfant était placé au centre du débat, ce dernier ne serait-il pas mieux éclairé ? (Bandelli 2019)

\section{Références}

Agasinski, S. (2013), Corps en miettes. Paris, Flammarion.

Allen, A.A. (2018), « Surrogacy and Limitations to Freedom of Contract: Toward Being More Fully Human ». Harvard Journal of Law and Public Policy, vol.41, $\mathrm{n}^{\circ 3}$, p.753-811.

Bandelli, D. (2019), « Feminism and Gestational Surrogacy: Theoretical Reconsiderations in the Name of the Child and the Woman ". Italian Sociological Review, vol.9, n`3, p.345-361.

https://doi.org/10.13136/isr.v9i3.297

Beeson, D., M. Darnovsky et A. Lippman (2015), « What's in a Name? Variations in Terminology of Third-party Reproduction ». Reproductive BioMedicine Online, $\mathrm{n}^{\circ} 31, \mathrm{p} .805-814$.

\section{https://doi.org/10.1016/j.rbmo.2015.09.004}

Brunetti-Pons, C. (resp.) (2017), Le «droit à l'enfant » et la filiation en France et dans le monde. Rapport final, CEJESCO de l'Université de Reims, Mission de recherche droit et justice, Convention de recherche n ${ }^{014.19}$, 5 janvier 2015-5 janvier 2017.

CEST (Commission de l'éthique de la science et de la technologie) (2009), Avis éthique et procréation assistée : des orientations pour le don de gamètes et d'embryons, la gestation pour autrui et le diagnostic préimplantatoire. Gouvernement du Québec.

Comité consultatif sur le droit de la famille (2015), Pour un droit de la famille adapté aux nouvelles réalités conjugales et familiales. Gouvernement du Québec.

https://www.justice.gouv.qc.ca

Conrad, P. (2007), The Medicalization of Society: On the Transformation of Human Conditions into Treatable Disorders. Baltimore, John Hopkins University Press. 
Corradi C. (2020), « Motherhood and the Contradictions of Feminism: Appraising Claims towards Emancipation in the Perspective of Surrogacy ». Current Sociology, vol.69, n², p.158-175.

https://doi.org/10.1177\%2F0011392120964910

CSF (Conseil du statut de la femme) (2016), Mères porteuses. Réflexions sur des enjeux actuels. Gouvernement du Québec.

https://csf.gouv.qc.ca/wpcontent/uploads/resume maternite pour autrui web.pdf

Côté, I., K. Lavoie et J. Courduriès (2018), « Penser la gestation pour autrui à partir des expériences vécues un ancrage empirique et multidisciplinaire ». Dans I. Côté, K. Lavoie et J. Courduriès (dir.), Perspectives internationales sur la gestation pour autrui. Expériences des personnes concernées et contextes d'action, Québec, Presses de l'Université du Québec, p.1-19.

Courduriès, J. (2016), “Ce que fabrique la gestation pour autrui ». Journal des anthropologues, nos144-145, p.53-76.

https://doi.org/10.4000/jda.6364

Decaluwe, B., A.-A. Poirier et G. Muckle (2016), « L'adoption coutumière chez les Inuit du Nunavik : ses spécificités et conséquences sur le développement de l'enfant ». Enfances Familles Générations, n²5.

http://journals.openedition.org/efg/1101

De Koninck, M. (2019), Maternité dérobée, mère porteuse et enfant sur commande. Montréal, Éditions MultiMondes.

Ekman, K.E. (2013), L'être et la marchandise. Prostitution, maternité de substitution et dissociation de soi. Ville Mont-Royal, M éditeur.

Épelboin, S. (2011), « Gestation pour autrui : une assistance médicale à la procréation comme les autres ? " L'information psychiatrique, vol.87, $n^{\circ} 7$, p.573579.

Feuillet-Liger, B. et M.-C. Crespo-Brauner (dir.) (2014), Les incidences de la biomédecine sur la parenté. Bruxelles, Bruylant.

Frison-Roche, M.-A. (2016), «Prohibition de la GPA : convergence absolue entre droits des femmes et droits des enfants ». MAFR blogue.

http://mafr.fr/fr/article/denoncer-la-strategie-des-industriels-de-lhumain-c/

Garrison, M. (2014), « La procréation médicalement assistée (PMA) aux ÉtatsUnis : un far-west de la filiation ». Dans B. Feuillet-Liger et M.-C. Crespo-Brauner (dir.), Les incidences de la biomédecine sur la parenté, Bruxelles, Bruylant, p.241-254. 
Godelier, M. (2014), "Systèmes de parenté et formes de famille ». Recherches de Science Religieuse, vol.102, n³, p.357-372.

https://www.cairn.info/revue-recherches-de-science-religieuse-2014-3-page$\underline{357 . h t m}$

Guilbault, D. (2018), « GPA. L'altruisme des femmes au service du marché ». Dans A.-L. Deram-Stoicea, M.-J. Devillers et C. Morin Le Sech (coord.), Pour le respect des femmes et des enfants, abolir la maternité de substitution, CIAMS, TheBookEdition.com, p.79-88.

Gross, M. (2018), «Pères gays et gestatrices ». Dans I. Côté, K. Lavoie et J. Courduriès (dir.), Perspectives internationales sur la gestation pour autrui. Expériences des personnes concernées et contextes d'action, Québec, Presses de l'Université du Québec, p.69-89.

Gunnarsson Payne, J. (2016), " Grammars of Kinship: Biological Motherhood and Assisted Reproduction in the Age of Epigenetics ». Signs - Journal of Women in Culture and Society, vol.41, n³, p.483-506.

https://doi.org/10.1086/684233

Harrison, L. (2014), « "I am the baby's real mother": Reproductive tourism, race, and the transnational construction of kinship ». Women's Studies International Forum, nº47, p.145-156.

https://doi.org/10.1016/i.wsif.2014.06.007

Héritier, F. (2010), « La filiation, état social ». La revue lacanienne, vol.3, n8, p.33-36.

https://doi.org/10.3917//rl.103.0033

HRC (Human Rights Council) (2018), Report of the Special Rapporteur on the sale and sexual exploitation of children, including child prostitution, child pornography and other child sexual abuse material, $37^{\mathrm{e}}$ session, 26 février-23 mars $(\mathrm{A} / \mathrm{HRC} / 37 / 60)$. https://ap.ohchr.org/documents/dpage e.aspx?m=102

lacub, M. (2004), L'empire du ventre. Pour une autre histoire de la maternité. Paris, Fayard.

Jacobson, H. (2016), The Labor of Love: Gestational Surrogacy and the Work of Making Babies. New Brunswick (NJ), Rutgers University Press.

Klein, R. (2017), Surrogacy: A Human Rights Violation. North Geelong (Australie), Spinifex Press.

Lafontaine, C. (2014), Le corps-marché. La marchandisation de la vie humaine à l'ère de la bioéconomie. Paris, Le Seuil. 
Lahl, J. (2018), «La vérité des grossesses à contrat : les contrats de maternité ne protègent ni les mères porteuses ni leurs enfants ". Dans A.-L. DeramStoicea, M.-J. Devillers et C. Morin Le Sech (coord.), Pour le respect des femmes et des enfants, abolir la maternité de substitution, CIAMS, TheBookEdition.com, p.89-95.

Le Breton, D. (2014), « La question anthropologique de la gestation pour autrui ». Dans B. Feuillet-Liger et M.-C. Crespo-Brauner (dir.), Les incidences de la biomédecine sur la parenté, Bruxelles, Bruylant, p.337-348.

Loulier, M. (2009), « Débat sur les mères porteuses : les stars qui assument et ouvrent la voie... ». Marie-Claire.

https://www.marieclaire.fr/,mere-porteuse-gestation-pour-autrui-people-sarahiessica-parker-polemique-avoir-des-enfants,20161,31254.asp

Noel, R. (2015), « Commercial Surrogacy and Materialist Feminism ». What's wrong?

https://whatswrongcvsp.com/2015/10/01/commercial-surrogacy-and-materialistfeminism/

PDF Québec (Pour les droits des femmes du Québec) (2017), « Enfantement pour autrui: esclavage des temps modernes ».

https://numerique.banq.qc.ca/patrimoine/details/52327/2786178

Ragone, H. (1994), Surrogate motherhood: Conception in the Heart. Boulder (CO), Westview Press.

Saravanan, S. (2018), A Transnational Feminist View of Surrogacy Biomarkets in India. Singapour, Springer Nature.

Segalen, M. (2017), "Why there can be no such thing as "ethical" surrogacy ». Travail, genre et sociétés, vol.2, n³8, p.53-73.

https ://doi.org/10.3917/tgs.038.0053\#xd co $\mathrm{f}=$ MDY5NzY1YTAtZTQxNi00N2YxL WJINjItMWMwN2M5YWJiMTg2

Surrogacy360.org (s.d.), « Current Law ». https://surrogacy360.org/considering-surrogacy/current-law/

Tourame, P. (2016), "Quelle liberté pour la mère porteuse ? " Les Cahiers de la Justice, n'2, p.275-288.

https://doi.org/10.3917/cdli.1602.0275 